\title{
Dynamic Rolling for a Modular Loop Robot
}

\author{
Jimmy Sastra Sachin Chitta Mark Yim \\ GRASP Laboratory, \\ University of Pennsylvania, \\ Philadelphia, Pennsylvania, USA \\ E-mail: $\{$ jsastra,sachinc,yim $\} @$ grasp.upenn.edu
}

\begin{abstract}
Reconfigurable modular robots have the ability to use different gaits and configurations to perform various tasks. A rolling gait is the fastest currently implemented gait available for traversal over level ground and shows dramatic improvements in efficiency. In this work, we analyze and implement a sensor-based feedback controller to achieve dynamic rolling for a loop robot. The robot senses its position relative to the ground and changes its shape as it rolls. This shape is such that its center of gravity is maintained to be in front of its contact point with the ground, so in effect the robot is continuously falling and thus accelerates forward. Using simulation and experimental results, we show how the desired shape can be varied to achieve higher terminal velocities. The highest velocity achieved in this work is 26 module lengths per second $(1.6 \mathrm{~m} / \mathrm{s})$ which is believed to be the fastest gait yet implemented for an untethered modular robot. One of the major findings is that more elongated shapes achieve higher terminal velocities than rounder shapes. We demonstrate that this trend holds going up as well as down inclines. We show that rounder shapes have lower specific resistance and are thus more energy efficient. The control scheme is scalable to an arbitrary number of modules, shown here using 8 to 14 modules.
\end{abstract}

\section{INTRODUCTION}

Locomotion is one of the most basic functions of mobile robots. Robotics researchers have demonstrated a wide variety of locomotion modes, including legged, wheeled, snake-like and even amoeba-like locomotion. Not every locomotion mode is suitable for all tasks. For example, a car like vehicle may be well suited for travelling over roads, but would not be suitable for climbing through a rubble pile doing search and rescue. A snake-like robot maybe well suited for climbing through tightly constrained environments, but is probably very inefficient going long distances.

Reconfigurable modular robots have been used as a platform to study different locomotion modes [24] and indeed, hundreds of locomotion modes have been demonstrated [1], [2], [3], [10], [20], [21], [22], [23]. The choice of configuration is usually task specific. One of the advantages of self-reconfigurable robots is the ability to reconfigure as the needs and environments change. For example, a self-reconfigurable robot in a search and rescue application might approach a scene using a fast and efficient rolling gait, reconfigure into a snake to squeeze through tight spaces and then reconfigure into a form with many limbs to move rubble or protect a victim. This paper will focus on a rolling loop configuration using a modular reconfigurable robot called СКВот [16].

In the loop configuration shown in Figure 1(a), the motion of the robot is like that of a tank tread. On flat terrain this gait is currently considered to be the most efficient as well as the fastest configuration under some conditions [20] (this has not been proven for the general case though). It has been implemented on various robots in [10], [18], [20]. In most implementations, the motion of these gaits was not dynamic, i.e. there was no inertial component to the motion. Rather the motions were purely kinematic; changes in geometry cause locomotion, stopping the changes in geometry also stop locomotion. There is a limit to the rate at which a kinematic rolling track can accelerate. Accelerating too fast causes the loop to undulate in place or worse roll backwards as shown in Figure 1(c).

In [9], Kamimura et al. implemented an open-loop dynamic rolling gait using CPGs where the weights for the CPGs were determined using simulation. A deformable robot was actuated by SMA coils in [18] to manipulate the shape into stable and unstable deformations for crawling and jumping. In [14], Matsuda and Murata proposed a robot whose links formed a closed chain where the actuators control the stiffness of a spring in each joints. This allows them to adjust the stiffness in each joint and drive the robot forward. In [17], a dynamic simulator was used to generate and simulate a dynamic rolling gait. Feedback was through accelerometers in the robot and an average velocity of about $1 \mathrm{~m} / \mathrm{s}$ was reported. However, this gait was not implemented on an actual robot and no analytical insight was provided. A dynamic rolling gait was implemented on a Icosahedral modular robot called the Tetrobot in simulation in [13]. However, no implementation details were given for this work.

In this work, we present a new implementation of the rolling loop using sensor-based feedback. Our work differs from previous work in the use of sensory feedback, development of a simplified dynamic model that provides considerable insight for development of control and implementation on a prototype robot. Sensory feedback dramatically improves the reliability of this gait (as compared to open loop implementations). In addition, this work presents the fastest gait yet reported by a modular robot.

The rolling loop is formed by a closed kinematic chain with many degrees of freedom. A complete model with all the joint degrees of freedom and the closed chain constraint for an arbitrary shape could be built for our robot, but the equations of 

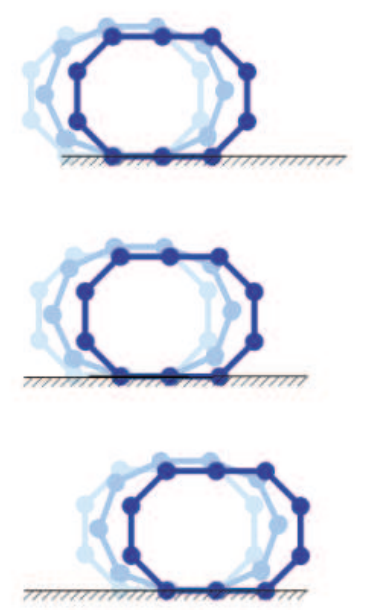

(a) Kinematic Rolling
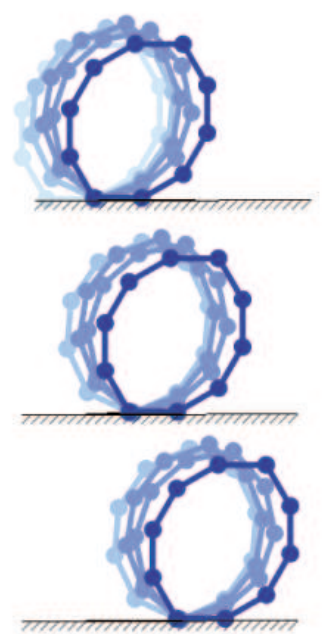

(b) Dynamic Rolling: Ideal Case
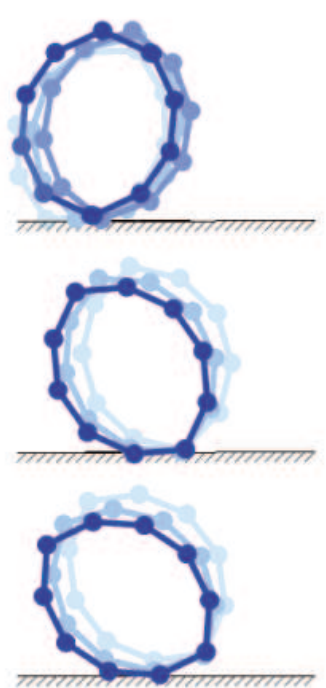

(c) Dynamic Rolling: Loop turns back on itself

Fig. 1. Different modes of rolling.

motion are very complex and would not provide much insight into the dynamics of the system. Further, with so many actuators on the robot, the dimension of the space of possible inputs makes designing controllers non-trivial. Our approach is to simplify the model for the system by restricting the type of controller to track an appropriate shape at touchdown, the contact of a module with the ground.

The resulting relatively simple controller gives us better insights into the dynamics of the system. Another benefit of this implementation is that the method scales to any number of modules or joints in a loop, within actuator limitations. In addition to simplifying the control algorithm, our approach also offers better insight into the dynamics of the system.

This paper is structured as follows. In Section II, we present the robot used in this work. In Section III, we introduce the main idea behind dynamic rolling and compare it with kinematic rolling. In Section IV we present a four-bar like model for the robot that simplifies the analysis of dynamic rolling. In Section V, we propose the framework used for control. The experimental setup is described in Section VI. In Section VII, we present theoretical results derived using this model and experimental results with 10 to 14 module rolling module loops. In Sections VIII and IX, we follow up with a discussion on insight gained from the results and possible future applications.

\section{THE ROBOT}

The robot system used in this work is a modular system called CKBOT and is shown in Figure 2. An individual module is shown in Figure 3. Each module is made up of a hobby servo that drives a rotary degree of freedom, a frame made of acrylonitrile-butadiene-styrene (ABS) plastic, a microprocessor and a touch sensor. A summary of the hardware is shown in Table I.

The one degree of freedom has a range of +90 to -90 degrees. When at 0 degrees, the module closely resembles a cube 60 $\mathrm{mm}$ on a side. As the degree of freedom moves away from zero, the rounded edge of the frame is exposed as in Figure 3. As a loop rolls, this rounded edge will make impact the ground. This rounded edge smooths rolling to some extent, though the top face of the module still poses a corner that impacts the ground.

Each module also has eight identical electrical connection ports, seven around four faces of the robot and one internal. These ports are used to electrically connect modules together as well as add extra computation, sensors or batteries. Power and communications are passed from module to module. Communication to each module is through a global bus based on the RoboticsBus protocol [7] which is built on the CANbus standard (Controller Area Network). 


\begin{tabular}{|c|c|}
\hline Property & Value \\
\hline Mass (per module) & $138(\mathrm{~g})$ \\
\hline Size (per module) & W60xL60xH60 $(\mathrm{mm})$ \\
\hline Batteries & Lithium Polymer 7.4V \\
\hline MCU & PIC18f2580 \\
\hline Servo & Airtronics 94359 \\
\hline Torque & $1.4 \mathrm{Nm}$ \\
\hline Reconfiguration & Manual \\
\hline
\end{tabular}

TABLE I

TECHNICAL SPECIFICATIONS FOR A CKBOT MODULE.

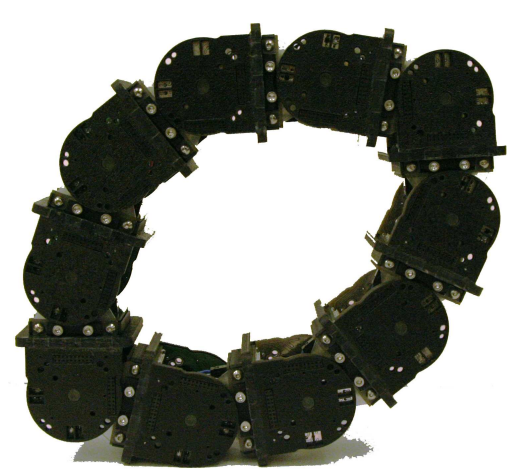

Fig. 2. Ten CКВот modules forming a football shape.

While each module is capable of carrying a battery, typically five lithium polymer battery packs were attached to a full loop during testing which would give several hours of run time. If long life performance were required, more batteries (up to 20) could be added to the system.

To form a loop, each module is attached end-to-end using screws and the two ends are then screwed together to form a loop. It is possible to form loops in other ways. For example, instead of daisy-chaining head to tail each module: (headtail)(head-tail)(head-tail) the modules could be attached head-head: (head-tail)(tail-head)(head-tail). This is the configuration that Superbot [2] and MTRAN [10] use. When tested with CKBOT, this configuration does not do as well in taking advantage of the rounded structure of the modules and thus the motion is not as smooth. As a result this was likely to be less efficient and was not tested extensively.

A separate microcontroller board, the brain, serves as a centralized controller. It plugs into one of the ports on the robot and provides control position commands for all modules. The touch sensors are infra-red proximity sensors that measure reflectance as an indication of distance to the ground. These touch sensors plug inside the module as shown in Figure 3 . Sensors use empirically derived thresholds for different surfaces to determine whether the module they are plugged into is touching down or not. The touch sensors send process messages to the brain upon a touchdown event. The brain then calculates the angles required for each module to track the desired shape and sends these commands to the microcontroller on each module.

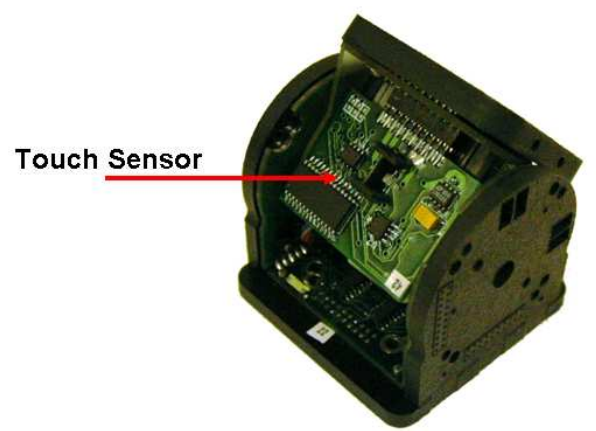

Fig. 3. An individual CKBOT module. 


\begin{tabular}{|c|c|c|c|c|c|c|c|c|c|}
\hline 60 & 60 & 60 & 0 & 0 & 60 & 60 & 60 & 0 & 0 \\
\hline 60 & 60 & 0 & 0 & 60 & 60 & 60 & 0 & 0 & 60 \\
\hline 60 & 0 & 0 & 60 & 60 & 60 & 0 & 0 & 60 & 60 \\
\hline 0 & 0 & 60 & 60 & 60 & 0 & 0 & 60 & 60 & 60 \\
\hline 0 & 60 & 60 & 60 & 0 & 0 & 60 & 60 & 60 & 0 \\
\hline
\end{tabular}

TABLE II

GAIT TABLE FOR KINEMATIC GAIT (ALL ANGLES ARE SPECIFIED IN DEGREES).

\section{Kinematic vs. DynAmic Rolling}

Statically stable locomotion is a term that is often used to characterize robot gaits. At any moment in a statically stable gait, the robot could stop moving its joints and the robot would not fall over. The projection of the center of gravity is always maintained to be within the convex hull of the ground contact points. Dynamic locomotion characterizes robot gaits in which the inertia of the robot plays an important role in the locomotion. In general, gaits (which are assumed to be stable) are either statically stable or dynamically stable, but not both. Traditionally static and dynamic stability refers to legged robot gaits. When applied to rolling gaits things become less clear. An automobile has its center of gravity always within the convex hull of its four tires. If it moves slow enough, the inertia of the vehicle can be ignored and it might be said that the vehicle is statically stable. However, if it gained any significant speed, the inertia cannot be ignored and the vehicle might be said to be dynamically stable. The line delineating the two conditions is not clear. In the case of loop robots, we refer to the gaits in which inertia plays no role as kinematic rolling. Here, the equations of motion can be determined directly from the geometry (no mass terms).

\section{A. Kinematic Rolling}

A kinematic rolling gait is implemented by repeatedly moving the shape of the loop such that the long axis rotates. This motion is similar to the motion of a tank tread. One rotation of the long axis corresponds to one cycle of the gait. The frequency of rotation is directly proportional to the speed, i.e. stopping the tread causes the whole robot to stop. For a closed loop robot like the one used in this work, one typical loop shape has two lines of modules one on top of the other attached by an intermediate set of modules forming arcs as shown in Figure 1(a). A kinematic roll for this configuration is executed by smoothly interpolating the joint angle of each module to the joint angle of the neighboring one in the loop. This type of motion can be easily represented using a gait table [20].

An example gait table for a kinematic rolling gait for a 10 module loop robot is shown in Table II. The neighboring columns of the table correspond to neighboring modules in the loop. The rows of the table correspond to steps (or time). The elements of the table are the joint angles for the corresponding module at the corresponding time. Note that there are only five rows in the gait table since the gait cycles back to the first row after the fifth.

One thing to note is that between rows only four modules change joint angles. This table can be scaled to larger numbers of modules by increasing the number of modules with 0 degrees (the straight parts). As the numbers get larger there would still be only four modules which change joint angles.

\section{B. Dynamic Rolling}

Unlike the kinematic gait a dynamic gait continues to move the robot even after all joints have stopped moving, i.e. a dynamic gait utilizes momentum. To create a dynamic rolling motion for a modular loop robot, one approach is to move the center of mass beyond the pivot point for the module currently on the ground as shown in Figure 1(b) and Figure 5. This results in a moment contribution from the weight of the robot in the direction of rolling and the robot accelerates in that direction.

The motion of the robot can be separated into two phases: (1) a shape change where the robot changes shape to the new desired shape that increases the distance between the center of mass and the ground contact point and (2) a falling phase where the robot's shape is frozen and the robot behaves essentially like an inverted pendulum pivoting about the contact point (bringing the center of mass closer to the ground contact point). The start of the first phase occurs as soon as a new touchdown is detected. This paper will show that the first phase results in a slight deceleration and then an acceleration while in the second phase the robot is continuously accelerating towards the next touchdown.

This motion is clearly not statically stable as the center of gravity is never within the convex hull of the ground contact points. One could say the robot is continuously falling. Since the robot is shaped like a loop, as long as it falls in the plane formed by the loop, it is never in a position where it cannot move (i.e. the way a legged robot may catastrophically fail if it falls over).

One way to view this method of control is that of a modified gait control table where the speed of motion between rows of a gait table is based on sensor feedback. 


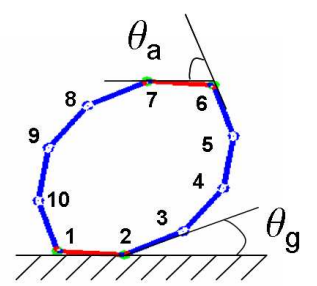

Fig. 4. Four-bar model used for analysis.

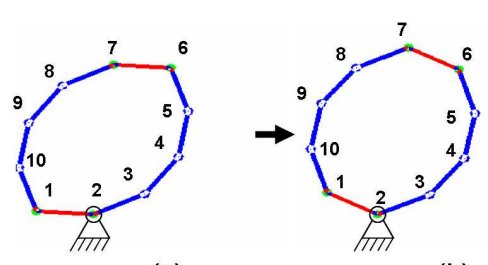

(a)

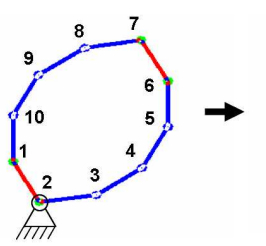

(d)

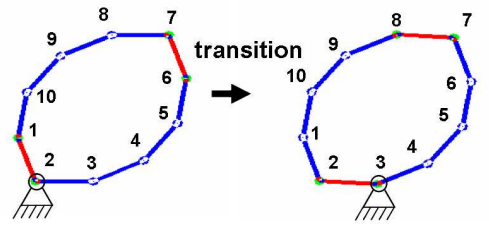

(e)

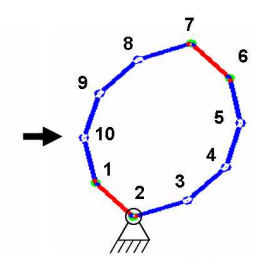

(c)

Fig. 5. Different phases of the rolling motion illustrating the effect of shape change at touchdown and subsequent falling motion of the robot.

\section{AnAlyticAl MODEL}

We make a simplifying assumption that serves to reduce the complexity of the dynamic analysis for the robot. We choose a "backbone" curve [4] to which we map the modules. We restrict this backbone curve to a shape that is formed by joining two equal arcs of a circle whose sector subtends an angle less than 180 degrees. This results in a shape which resembles an American football as shown in Figure 2. In the limit, as the two arcs approach 180, the shape reduces to a circle. The modules of the robot approximate this backbone curve by fitting the position of the joints to lie on the arcs.

The shape can be defined using a single parameter $\theta_{a}$, the angle between the modules at the top and bottom apex of the shape (Figure 4). All the other joint angles are equal to each other (to say $\theta_{s}$ ) and can be derived in terms of $\theta_{a}$ from Equation 1

$$
2 \theta_{a}+(n-2) \theta_{s}=2 \pi
$$

where $n$ is the number of modules in the loop.

It will be clear from our choice of control strategy in Section $\mathrm{V}$ that local shape changes of the robot will involve only four modules moving at a time just as the kinematic gaits in Section III-A. We can thus simplify the model of the loop to that of a floating four-bar mechanism hinged at the contact point. In this model the four moving modules represent the four joints. The two longer arcs (nodes 2 through 5 and nodes 7 through 10) represent two of the links of the four-bar while the other two links (comprising node 1 and 6) are made up of single modules. Reducing the model in this manner to the one degree of freedom four bar linkage means that the shape of the robot can be parameterized using a single parameter, $\theta_{a}$ (or similarly $\theta_{s}$ ). This framework is shown in Figure 4.

The equations of motion for this simplified version of the robot are derived using a standard method by first defining the Lagrangian for the system and deriving Lagrange's equations. The generalized coordinates used in the analysis are the apex angle $\theta_{a}$ and the global angle made by the robot with the ground $\theta_{g}$ (Figure 4). Each module is considered to be a thin rod of length $0.06 \mathrm{~m}$ with mass $0.138 \mathrm{~kg}$ (from Table I). The resultant equation for the evolution of $\theta_{g}$ can be expressed in the form:

$$
\ddot{\theta}_{g}=f_{1}\left(\theta_{a}, \theta_{g}\right) m g+f_{2}\left(\theta_{a}, \theta_{g}\right) \tau \text {. }
$$

where $m$ is the mass of a module and $g$ is gravity. Note that the first term on the right hand side essentially collects the terms that are linear in $m g$ while the second term on the right hand side collects all the terms linear in $\tau$. $f_{1}$ and $f_{2}$ are functions 

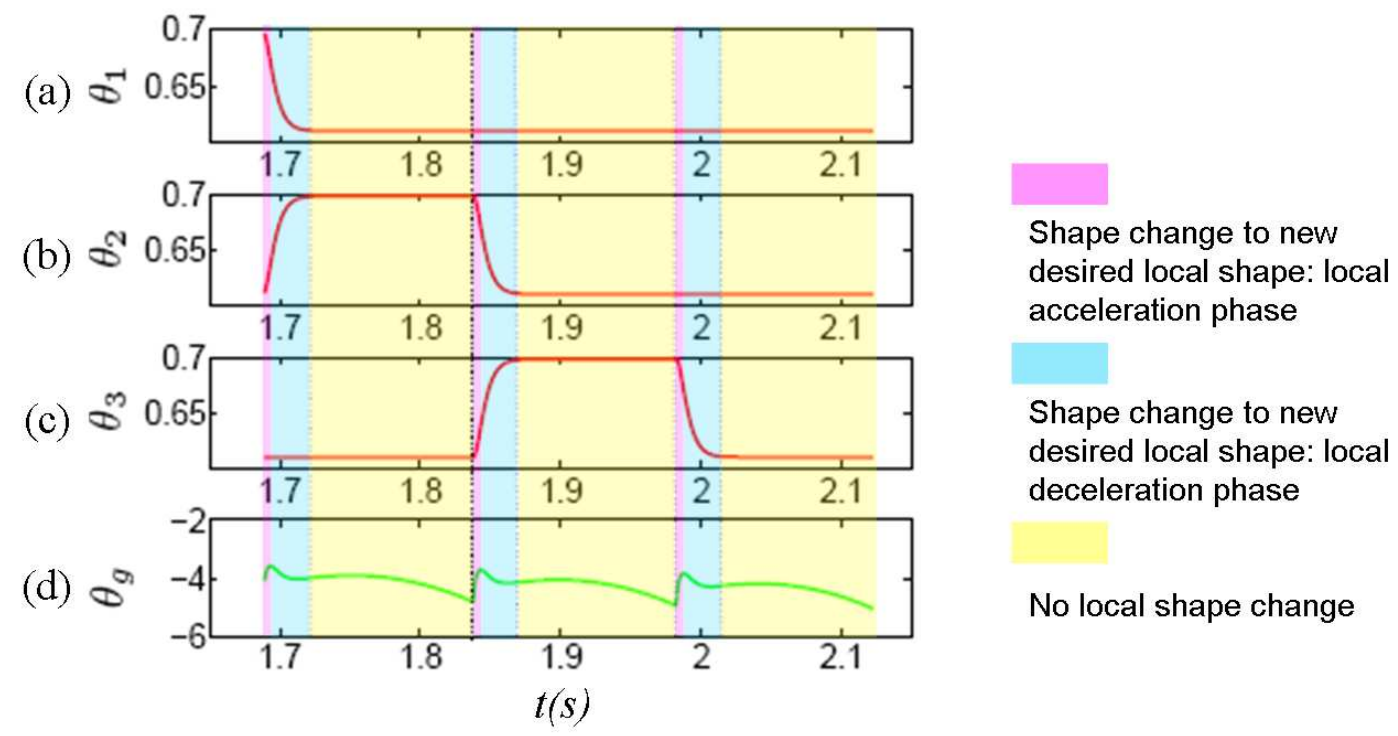

Fig. 6. (a) Joint angle for Module 1 (b) Joint angle for Module 2 (c) Joint angle for Module 3 (d) Angular velocity of the robot over time interval corresponding to three consecutive module touchdowns.

of the two angles $\theta_{a}$ and $\theta_{g}$ and constant parameters including the length of the module $l$, its mass $m$ and the mass moment of inertia $\left(I_{0}\right)$ of the module about its rotational degree of freedom. $f_{1}$ and $f_{2}$ are presented in detail in the Appendix.

Equation 2 shows that there are two contributing terms to the angular acceleration of the robot: (1) the moment due to gravity about the point of contact with the ground and (2) a coupling term arising from the coupling of $\theta_{a}$ and $\theta_{g}$. The direction of the coupling term is initially against the direction of rolling, while the moment arm due to gravity is always towards the direction of rolling. Thus, the robot first decelerates during the beginning of the shape change phase (as shown by the pink part of the graphs in Figure 6) and then accelerates due to a change in sign of the coupling term in the remaining part of the shape change phase (shown in blue in Figure 6). After finishing the shape change phase, it then continuously accelerates in the free-fall phase (shown in yellow in Figure 6) solely due to the influence of the moment-arm due to gravity. As the robot rolls faster, the duration of the free-fall phase gets shorter. Beyond a certain speed, it is possible that the robot is unable to go through its complete shape change before touchdown in which case the simplified four-bar model we use in our work is no longer valid. This corresponds to touchdown happening at a time before the yellow region in Figure 6. Since our model is no longer valid in these cases, we do not report or use these results for further analysis.

We define a step of the gait as the sequence of events between consecutive touchdowns of two adjacent modules. At touchdown, we reassign the nodes to the different links based on the global positions of the nodes. This is illustrated in Figure 5. In Figure 5(a) joints at 1, 2, 6 and 7 form the joints of the four-bar linkage. After the transition to Figure 5(f) the four bar is represented by the joints 2, 3, 7 and 8 and joint 3 becomes the pivot point around which the fourbar linkage is hinged.

When the module comes into contact with the ground, a transition condition is defined at impact of the module on the ground. Joint angles and the position of the robot stay fixed at transition while velocities are transformed using the transition conditions. The transition condition relates the angular momentum $L_{-}$of the whole body of the robot about the new pivot point on the ground just before impact with the angular momentum $L_{+}$of the whole body about the new pivot point after impact. Using a coefficient of restitution $\eta$ (found empirically to be 0.94 ), the transition condition is given by the momentum transfer equation 3 on impact.

$$
L_{+}=\eta L_{-} .
$$

Thus, at each step energy is lost with each impact based on $\eta$. Also at each step energy is input to the system by the motors as the loop changes shape. The energy input, to a first order, is constant with each step, however, the energy lost is a function of velocity (as a component of momentum). So, it is logical to propose that as the system accelerates from zero velocity, a terminal velocity will be reached where the energy input to the system is equal to the energy lost, assuming a stable steady state. 


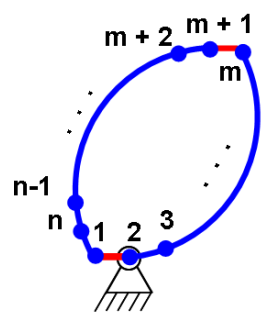

(a)

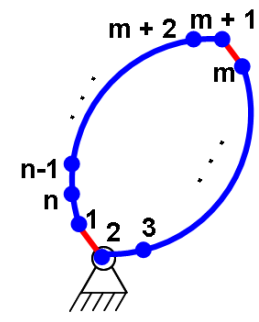

(d)

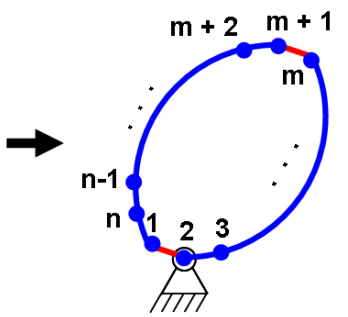

(b)

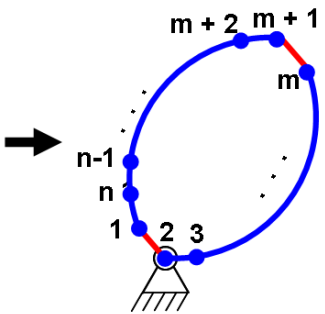

(c)

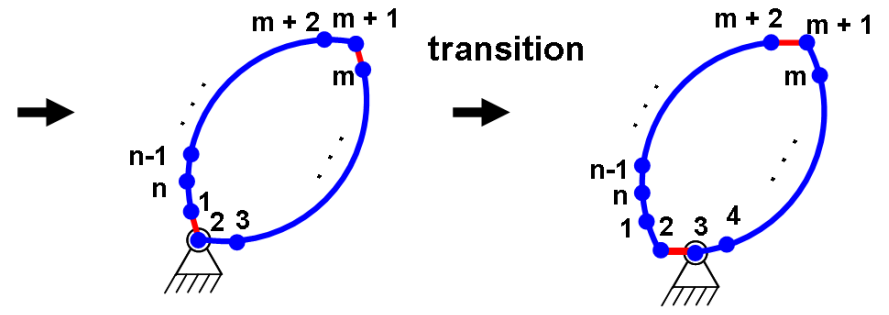

(e)

(f)

Fig. 7. Scalability of the controller to different number of modules.

\section{A. Scalability}

The particular choice of parameterization made for the controller earlier in Section $\mathrm{V}$ has the advantage of making the controller easily scaleable to configurations with a different number of modules. This has an advantage in designing controllers for modular robots since it reduces design and computational requirements for control and makes the controller invariant to the number of modules in the loop. Consider, for example, Figure 7 where a loop robot with $n$ modules is shown. In Figure 7(a), the apex nodes are 1 and $m$ where $m=1+n / 2$. The link joining nodes 1 and 2 (Link 1 ) and the link joining nodes $m$ and $m+1$ (Link 2) form two links of the four-bar used for analysis and control. The third link (Link 3 ) is formed by a combination of the links joining joints 2 through $m$ and the fourth link (Link 4) is formed by a combination of the links joining joints $m+1$ through $n$ and 1. Thus, a multi-degree of freedom rolling loop with $n$ modules can be reduced to the same four-bar linkage used for analysis.

Note that all the joints in Link 3 and Link 4 are stationary during the shape change phase and the only joints that move are the 4 joints that attach Link 1 and Link 2 to Link 3 and Link 4. The control scheme relies on position control of the servos to maintain the shape of Links 3 and 4, even though they are not moving there is some power consumed to maintain this shape. As the number of modules in these links get larger the weight of the modules will cause larger draws on power, even exceeding the torque limits of the actuators. One interesting property is that at higher speeds, centrifugal forces will counteract gravity reducing torque requirements and saving energy.

The scalability of the controller to different number of modules was tested by implementing the controller on rolling loop configurations with $8,10,12$ and 14 modules.

\section{CONTROL}

In Section IV, we made a simplifying assumption that allowed parameterization of the desired shape at touchdown using a single parameter, the apex angle $\theta_{a}$. The controller used for dynamic rolling can now be described by specifying a new desired shape for the robot at touchdown such that the robot is falling forwards with respect to the pivot point describing the contact of the robot with the ground. This corresponds to designating node 7 and 2 in Figure 5(a) as the new apex angles of the shape. When a new desired shape is specified the loop changes shape as is illustrated in Figure 5(b)-(c)-(d). Once it reaches the new desired position, the local shape does not deform anymore. Now, the robot undergoes a pure falling motion (Figure 5(d)). The robot falls like an inverted pendulum until node 3 touches down on the ground (Figure 5(e)).

Shapes that are more elongated (corresponding to higher values of $\theta_{a}$ ) will result in a larger moment arm and higher angular acceleration. However, the amount of shape change (represented by the net change of joint angles) is also higher. Rounder shapes correspond to a smaller value of $\theta_{a}$ and will result in a smaller moment arm and smaller amount of shape change. Figure 8 shows the effect of the choice of shape on the moment arm due to gravity. Figure 8(a) shows the smaller moment 


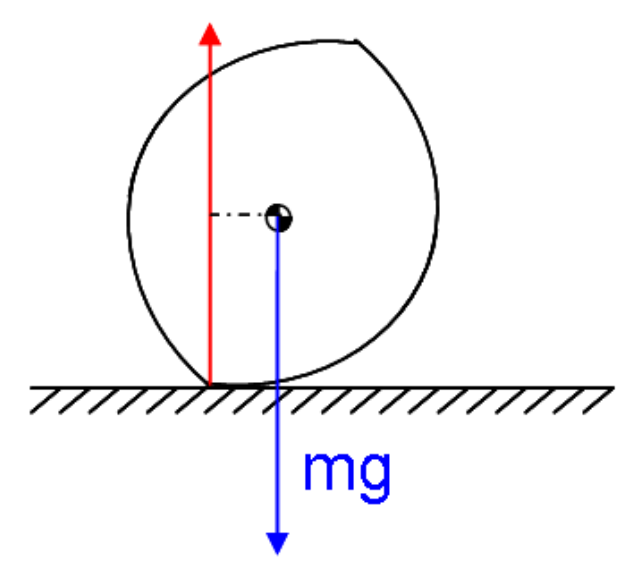

(a) Moment arm corresponding to a rounder shape at (b) Moment arm corresponding to a more elongated shape touchdown.

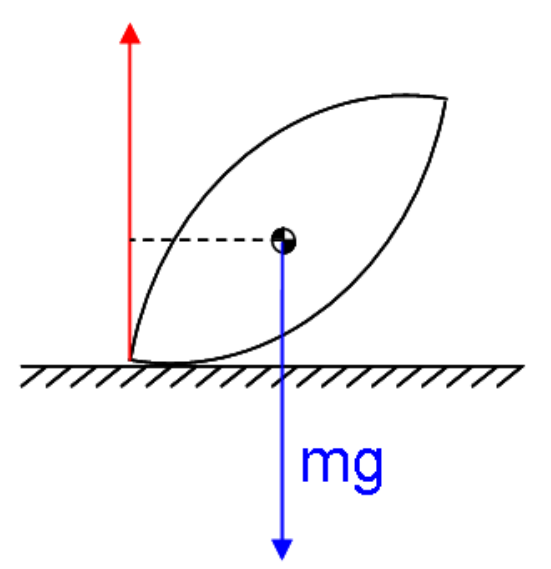

at touchdown.

Fig. 8. Effect of shape on moment arm at touchdown.

arm corresponding to a rounder shape and Figure 8(b) shows the larger moment arm corresponding to a more elongated shape. We should expect that more elongated shapes will give us higher accelerations while rounder shapes may be more efficient. We will examine the effect of the desired shape on the speed of the robot by varying the parameter $\left(\theta_{a}\right)$.

This shape control is implemented by the brain board sending the angular positions to corresponding modules over the RoboticsBus at $60 \mathrm{~Hz}$. Each microcontroller on each module generate PWM signals to the servos which then use a highly tuned PID position control to maintain or attain the commanded position.

\section{EXPERIMENTS}

\section{A. Terminal velocity and specific resistance}

One of the main objectives for the experiments is to see if the trends proposed from the analysis of the model hold true, namely

1) The robot achieves a terminal speed during rolling and this speed increases with increase in $\theta_{a}$.

2) Rounder shapes are more efficient.

Reflective markers were placed on the robot to track a single module and its joint angle by a high speed motion capture system (VICON). Measurements were recorded at a speed of $100 \mathrm{~Hz}$ and a resolution of $0.1 \mathrm{~mm}$. The overall workspace of the VICON however was limited to $3 \mathrm{~m} \times 3 \mathrm{~m}$. To determine the terminal velocity the position of the robot was measured manually from video to increase the available workspace. Each trial to determine the terminal velocity consisted of two parts. In the first part a running start of $4 \mathrm{~m}$ was given to the robot to allow it to reach terminal velocity. No measurements were taken in this part. In the second part, the robot would continue rolling and position was determined manually from the video footage by marking time stamps as the robot crosses markings on the carpet spaced at 1 foot intervals. The field of view of the camera covered only the second part of the trial.

The desired shape of the robot was specified using the parameter $\theta_{a}$ for a 10 module robot. $\theta_{a}$ was varied between $36^{0}$ to $90^{0}$. $\theta_{a}=36^{0}$ represents a shape where $\theta_{a}=\theta_{s}$ and there is no change in the shape of the robot while $\theta_{a}=\pi / 2$ represents an elongated shape where the amount of shape change in the robot between touchdowns will be very high. It was found that shapes with $\left(\theta_{a}\right)$ greater than $70^{\circ}$ could not be tracked accurately by the controller. However, results for these values are still reported here.

Specific resistance $(\epsilon)$ measures the energy cost of locomotion per unit distance and robot weight. It is calculated as follows:

$$
\epsilon=\frac{P}{m g v}
$$

where $P$ is the power input to the robot, $m$ is the total mass of the robot, $g$ is the acceleration due to gravity and $v$ is the average speed of the robot. Specific resistance is a natural measure for the second claim above, i.e. that rounder shapes consume less power.

Experimentally measuring specific resistance requires the measurement of the power consumed by the robot and the average speed achieved by the robot over the corresponding run. A robot with 10 modules and 5 lithium polymer batteries has a mass of $1.7 \mathrm{~kg}$. Normalizing the power consumption in this manner with respect to both the speed and mass of the robot allows meaningful comparison between robots of different sizes and speeds. 


\section{B. Motion on inclines}

Our initial studies showed that the robot works well on level terrain, but for this gait to be really useful in space exploration, search and rescue or any real world scenario we wish to show that it behaves well on non-flat terrain as well. Examining traversal on an inclined terrain is a step towards more unstructured terrain. It should be obvious that rolling down an incline is possible (e.g. just by maintaining a circular shape) however, traversing up is not as clear. Experiments were performed going up a slope on an incline of 5 degrees and down a sleep with an incline of -5 degrees. Rolling motion up a long incline is a good measure of the robustness of the controller to a constant source of disturbance while rolling down an incline tests the controller's ability to react to faster touchdown events.

Terminal velocities and power consumption were measured and compared with behavior on level terrain. Multiple trials were carried out for each shape and incline on the same carpet to maintain consistency across trials.

\section{Scalability of the controller}

As noted earlier, the particular parameterization chosen for the rolling loop makes it easier to scale the controller to loops with different number of modules. This was tested by implementing the controller on rolling loop configurations with 8,10 , 12 and 14 modules. The controller maps the links and joints of all these configurations onto the four-bar like backbone curve used earlier for analysis. The user chooses the value of $\theta_{a}$ and $\theta_{s}$ can be easily determined from $\theta_{a}$ using Equation 1 . The controller then designates the module touching the ground and the one diameterically opposite as the apex and sets all other joint angles to a constant value of $\theta_{s}$. The control algorithm for $n$ modules is thus the same as the one used for the loop with 10 modules. This demonstrates the versatility and scalability of the controller.

\section{Speed control}

Experiments were also carried out to demonstrate arbitary speed control with a human specifying the desired speed using a joystick in real time. The robot could be sped up by increasing the apex angle specified by the controller. Braking motion to slow the robot was achieved by designating the module in front of the current touchdown module as the apex of the new desired shape.

\section{E. Experimental setup}

Experiments were carried out on multiple surfaces, but the results reported here are for carpet flooring. The choice of surface on which the robot rolls has a visible effect on the speed of the robot. The robot was slower on thick carpet than on a thin carpet placed on a marble floor where the fastest run times were achieved. The choice of flooring also affects the performance of the IR touch sensors. Thresholds for the sensors were set manually on different floor surfaces to achieve the best performance.

Ground truth data was provided by the high speed motion capture system (VICON). The VICON motion capture system provides measurement of pose of one of the modules and one joint angle of the robot at a high speed (100 Hz) and submillimeter accuracy. This allowed comparison between the actual and desired trajectories of the joints on the robot and let us verify that the controller triggers the correct module on touchdown at the correct time.

\section{RESULTS}

\section{A. Tracking of desired joint trajectories}

Figure 9 plots experimental tracking results for one of the joint angles of the robot and also the global position of the robot. Figure 9(a) shows the height of one of the modules and it should be noted that the crests in the $z$ positions in Figure 9 represent touchdowns for the module diametrically opposite the tracked module while the troughs represent touchdowns for the tracked modules themselves. The joint angle of this module is shown in the middle figure and we can verify that the module reaches $\theta_{a}=\pi / 4$ and goes back to $\pi / 6$ and that this motion is triggered upon touchdown of the module i.e. when the $z$ position is at a minimum. Note that the duration where the module holds the apex angle $\theta_{a}$ is very short.

\section{B. Terminal velocity and specific resistance}

Figure 10 plots simulation and experimental results for the final speed of the robot for different desired shapes at touchdown. As predicted in the analysis of the model, the observed behavior of the system was that a terminal velocity was reached. In addition, as the desired shape becomes more and more elongated (corresponding to increase in the value of $\theta_{a}$ ), the terminal velocity achieved by the robot increases. Also as the desired shape grows elongated, the angular acceleration of the robot in its free fall phase also increases thus resulting in a higher terminal speed. Shapes with an apex angle greater than $70^{\circ}$ cannot keep up with the speed because the servo cannot move fast enough to reach the next shape before the next touchdown.

Below a certain magnitude of shape change, the robot has no terminal velocity. When $\theta_{a}=\theta_{\text {critical }}$ the robot has just enough energy to continue motion. Note in the continuous case (with infinite modules) the terminal velocity at $\theta_{\text {critical }}$ approaches 0 . Geometric observation shows that when $\theta_{a}=37^{\circ}$, the center of gravity sits over the new touchdown point. In simulation, 


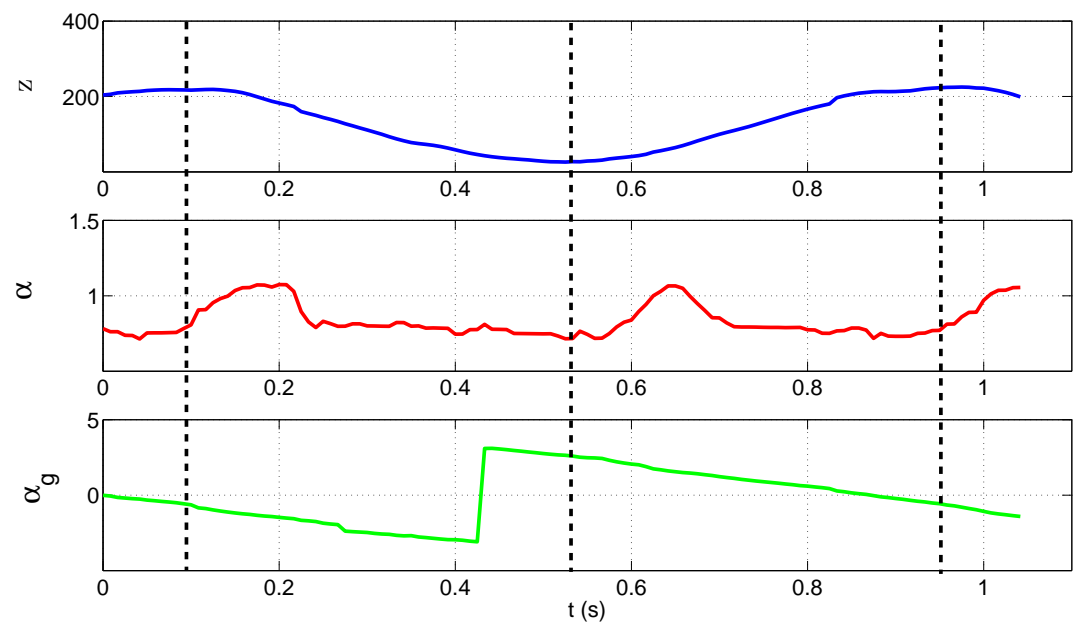

Fig. 9. Experimental results: Tracking results for one module using a motion capture system: $z$ represents height of tracked module above the ground for one cycle, $\alpha$ represents joint angle of tracked module, $\alpha_{g}$ represents pitch of tracked module with respect to global reference frame (the discontinuity in the data is because of a jump from $-\pi$ to $\pi$ ).

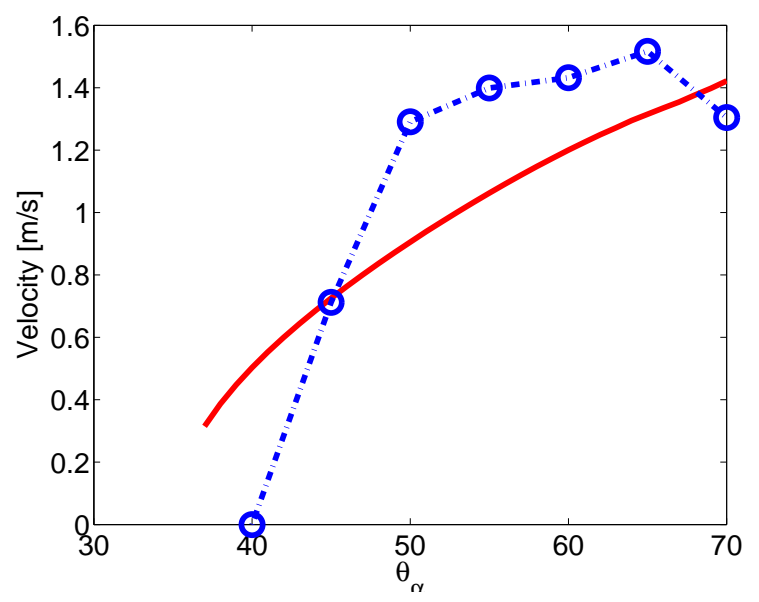

Fig. 10. Terminal velocity vs. $\theta_{a}$. Simulation shown by a solid line and experimental results by a dotted line (with (o)'s).

with values of $\theta_{a}$ less than $37^{\circ}$, the robot slows down to a halt even if it has some initial momentum. For $\theta_{a} \geq 37^{\circ}$, the robot is able to sustain its momentum in simulation and roll continuously. Experimentally, the robot does not achieve continuous motion unless $\theta_{a}>40^{\circ}$. The experimental terminal velocities are close to the predicted velocities.

Figure 11 plots simulation and experimental values for the specific resistance for different desired shapes. The power determined analytically should be lower than the actual power input to the robot, because the simulation only takes into account the power used to change shape. This is shown in our results. The experimental measurements show larger specific resistance than the theoretical measurements in all trials. More importantly the trend stays the same, i.e. rounder shapes exhibit lower specific resistance and are more efficient.

Another estimate of energy efficiency of a gait is the amount of travel in joint space that each module must move in order to move forward. This is measured by the difference between the two angles $\theta_{a}-\theta_{s}$. By this measure, rounder shapes also use less energy than the more elongated ones as $\theta_{a}-\theta_{s}$ is smaller. It is worthwhile noting that, based on this measure, dynamic gaits with rounder shapes are also more efficient than kinematic gaits. Maintaining any velocity using a purely kinematic gait typically requires a large traversal of modules in joint space while, once some speed has been built up, dynamic gaits can be sustained using smaller effort in joint space.

To compare these numbers with those for a kinematic gait, specific resistances for different dynamic rolling gaits as well as kinematic rolling gaits are plotted against terminal velocity as shown in Figure 12. The kinematic rolling gait has higher specific resistance than all the dynamic rolling gaits which implies that the amount of energy used to move a unit distance is lower in dynamic rolling than in kinematic rolling, which is what one would expect. For completeness, the electrical power consumed by the total robot is presented in Table III for the kinematic as well as the dynamic gait. 


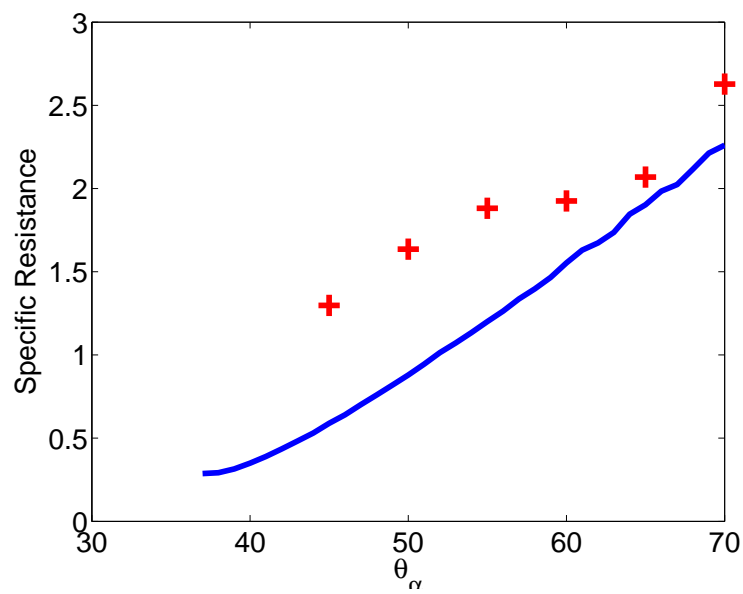

Fig. 11. Specific resistance vs. $\theta_{a}$. Simulation results shown with a solid line and experimental results with a ' + '.

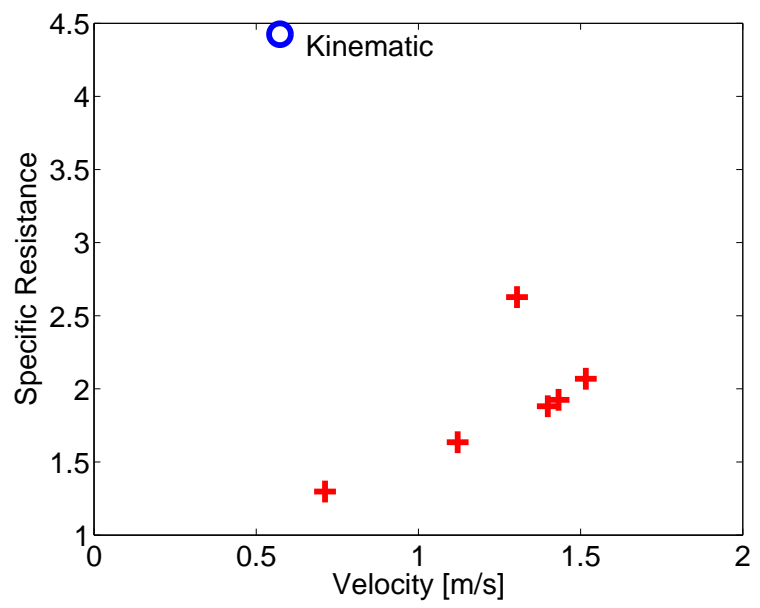

Fig. 12. Experimental results: specific resistance vs. velocity. Dynamic rolling (shown with '+') and kinematic rolling (shown with 'o').

\section{Motion on inclines}

Figure 13 summarizes the terminal velocities of the robot in a dynamic rolling gait on different inclines $\left(-5^{0}, 0^{0}\right.$, and $\left.5^{0}\right)$. On each incline, values of $\theta_{a}$ between $36^{0}$ to $70^{\circ}$ were used.

In the case of a downward slope, rolling motion with a terminal velocity of $0.9 \mathrm{~m} / \mathrm{s}$ was achieved even for $\theta_{a}=36^{0}$ while on level terrain no motion was achieved for $\theta_{a}<=40$ degrees. On the upward slope no motion was achieved for $\theta_{a}<=50^{0}$. The trend of terminal velocity increasing with more elongated shapes is preserved on all the inclines. The terminal velocity also saturates at a lower value for higher slopes of the terrain.

\section{Scalability}

Figure 14 shows that terminal velocity increases logarithmically with an increase of 3.7 times between 8 and 10 modules. There is only a small increase found between 10 and 12 modules and no significant difference between 12 and 14 modules.

\begin{tabular}{|l|l|}
\hline 45 & 1.541 \\
\hline 50 & 3.057 \\
\hline 55 & 4.389 \\
\hline 60 & 4.597 \\
\hline 65 & 5.231 \\
\hline 70 & 5.715 \\
\hline Kinematic & 4.232 \\
\hline
\end{tabular}

TABLE III

Apex Angle (IN DEGReEs) Vs. PoWer (IN WATtS) 


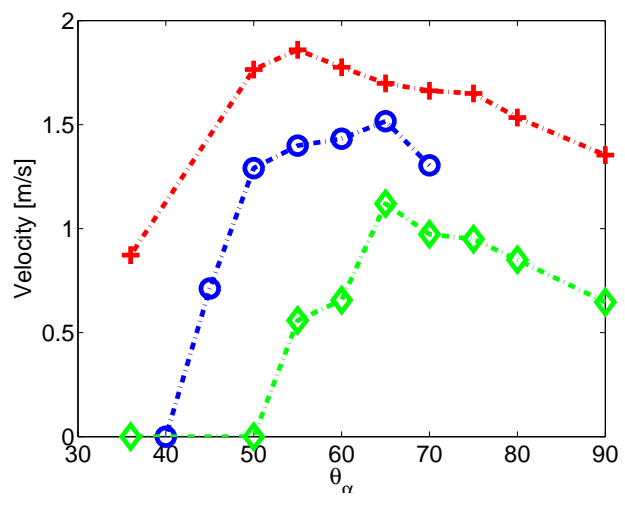

Fig. 13. Experimental results: specific resistance vs. velocity on $5^{\circ}$ (shown with '+'), $0^{\circ}$ (shown with 'o') and $-5^{\circ}($ shown with ' $\diamond$ ) inclines.

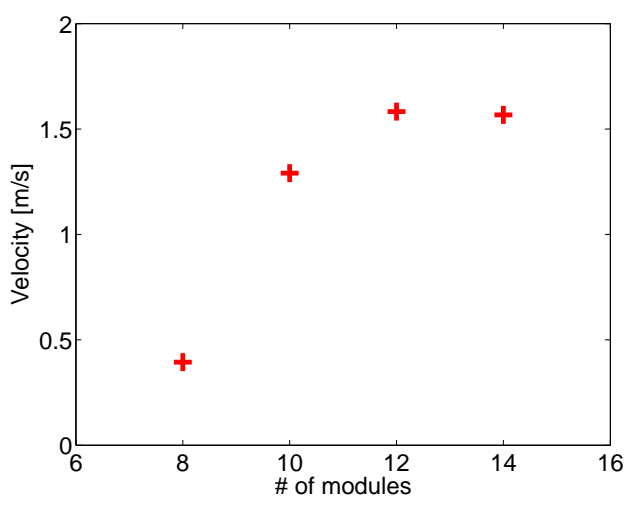

Fig. 14. Terminal velocity vs. number of modules. Apex angle was 50 degrees in all experiments.

The terminal velocity saturates and approaches a limit at $1.6 \mathrm{~m} / \mathrm{s}$.

To compare terminal velocity between the loop of 8 and the loop of 10 modules we can scale the terminal velocity by dividing by the length of the loop. For the case with 8 modules, this corresponds to a speed of $0.4 /(8 \times 0.06)=0.833$ loop lengths per second, while for the case with 10 modules this corresponds to a speed of $1.29 /(10 \times 0.06)=2.15$ loop lengths per second. For configurations with 12 and 14 modules, the speeds in loop lengths per second are smaller than for the case of 10 modules since the weight of the robot plays a more significant role. It is harder to maintain or change the shape of a robot with more number of modules.

This number is a measure of speed that accounts for the difference in size of the loops and shows that a loop with more joints has a higher velocity in terms of loop lengths per second. A loop with more joints can more accurately accurately approximates the arc of the shape. Thus, these results show that if the arc is more accurately approximated the faster the gait is.

In a loop of 14 modules the servos had enough torque to maintain its shape however saturation still occurred. This could be explained by limitations of the touch sensors that operate at $60 \mathrm{~Hz}$ and speed of the servos when changing shape. There were no issues with stability on smooth level terrain in the transverse plane with the larger or smaller loops.

\section{DISCUSSION}

One of the major findings of this work is that elongated desired shapes at touchdown for a rolling loop lead to higher terminal velocities. This is shown through a combination of simulation and experiments. The result makes sense intuitively as more elongated shapes create a larger moment arm due to the center of gravity w.r.t. the ground contact point. Because of this greater moment more acceleration occurs in the falling motion and more energy is put into the system at each step, a result that agrees with our theoretical predictions. It is interesting to note that the acceleration phase of the dynamic gait is similar to the motion of an inverted pendulum which has been shown in the context of walking to be very efficient requiring no work input to move the center of mass [12]. The fastest experimental gait had a speed of $1.6 \mathrm{~m} / \mathrm{s}$ (roughly 5.4 body lengths per second for the 10 module robot.) Since the robot can reconfigure to different bodies lengths and use different gaits for different applications, a more apt measure for speed may be to normalize to module size. Using this measure the $1.6 \mathrm{~m} / \mathrm{s}$ translates to 26 module lengths per second. To the authors' knowledge this is the fastest gait for any untethered modular robot. 

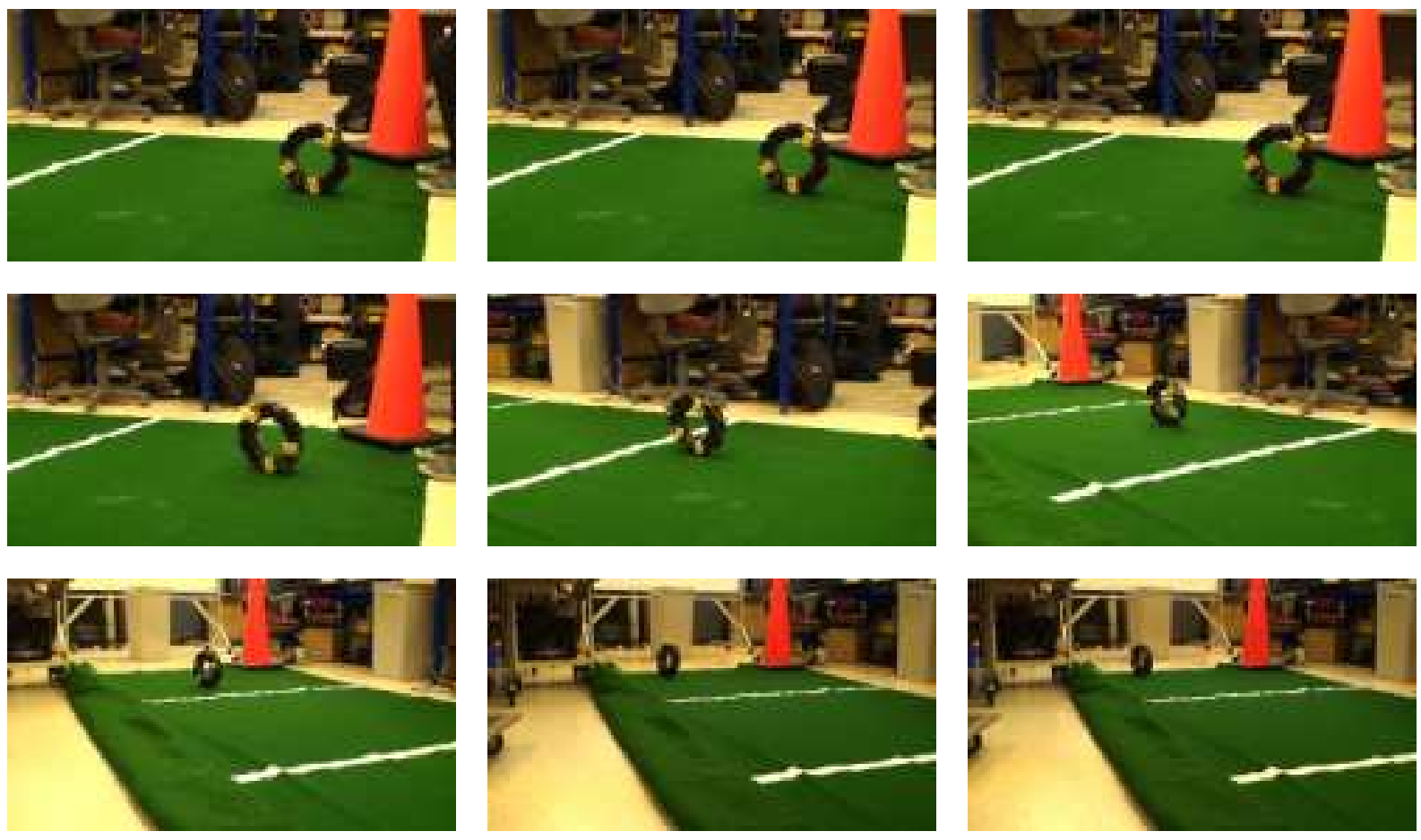

Fig. 15. Snapshots of the rolling motion.

Although the experimental and the theoretical results for the terminal velocity are close, the experimental results are consistently lower. There may be several possible reasons for this difference between the predicted and the actual behavior. One of the main reasons is that we have not taken into account friction in the modules and did not build a motor model for the servos. Our model also assumes that the modules can be represented as rods (for determination of inertia parameters). The actual modules however have a complex shape that could have different moment of inertias. Also, the contact point is not an ideal hinge point. An individual module has a more complex shape and comes into contact with the ground at more than one point. Therefore contact dynamics would be a good place for improvement in the model.

The terminal velocities saturated for desired shapes with a high apex angle. Hardware limitations in the current prototype may be partly responsible for this saturation. At a speed of $1.6 \mathrm{~m} / \mathrm{s}$, touchdowns occur at about $27 \mathrm{~Hz}$ and the hobby servos used in the prototype are unable to track the desired shape changes for speeds higher than this. Limited bandwidth on the communications bus might be another reason for this saturation. We have observed frames representing touchdown being dropped by the controller which could result in the controller's inability to keep up with the desired shape changes.

Conversely, for smaller values of $\theta_{a}$, the controller was unable to initiate motion in the robot. The desired shape needs to move through a certain angle for the center of gravity of the resultant shape to lie outside the base of support formed by the module on the ground. Thus, motion is only initiated after overcoming this initial load.

In simulation and experiments, we also show that although more elongated shapes lead to higher terminal velocities, rounder shapes have lower specific resistance. This means that more elongated shapes are less energy efficient. The result makes sense intuitively as rounder shapes need to travel less distance in joint space at each step. We believe higher rolling speeds should be more energy efficient (as modules on the top do not need to fight gravity due to the centrifugal force).

While the most efficient gait may be the roundest one, it is also the slowest to accelerate. One strategy for faster yet still more efficient rolling is to start with an elongated shape to accelerate quickly, then decrease $\theta_{a}$ linearly with speed until $\theta_{a}=\theta_{s}$. As it gets faster the shape becomes less oval and more circular. At the limit the shape will be that of a perfect circle which will roll using zero energy.

Discrepancy between theoretical and experimental specific resistances are due to limitations in the model as explained earlier. Additionally, the analytical power computed only takes into account the power used by modules that are moving. It does not take into account the power used by modules that do not change their joint angle. However, the critical result to note here is that the trend in variation of specific resistance found through experiments with change in the desired shape matches the trend found through simulation.

Figure 16 shows an interesting comparison between the specific resistance of the dynamic rolling gait of CKBOT, other gaits for CKBOT (including a kinematic rolling gait, a crawling gait and an inchworm gait) and other robotic systems with non-wheeled modes of locomotion like walking. Here, all quantities have been plotted on a logarithmic scale. Ideally, we would like to have as low a specific resistance as possible at as high a speed as possible. This corresponds to being on the 


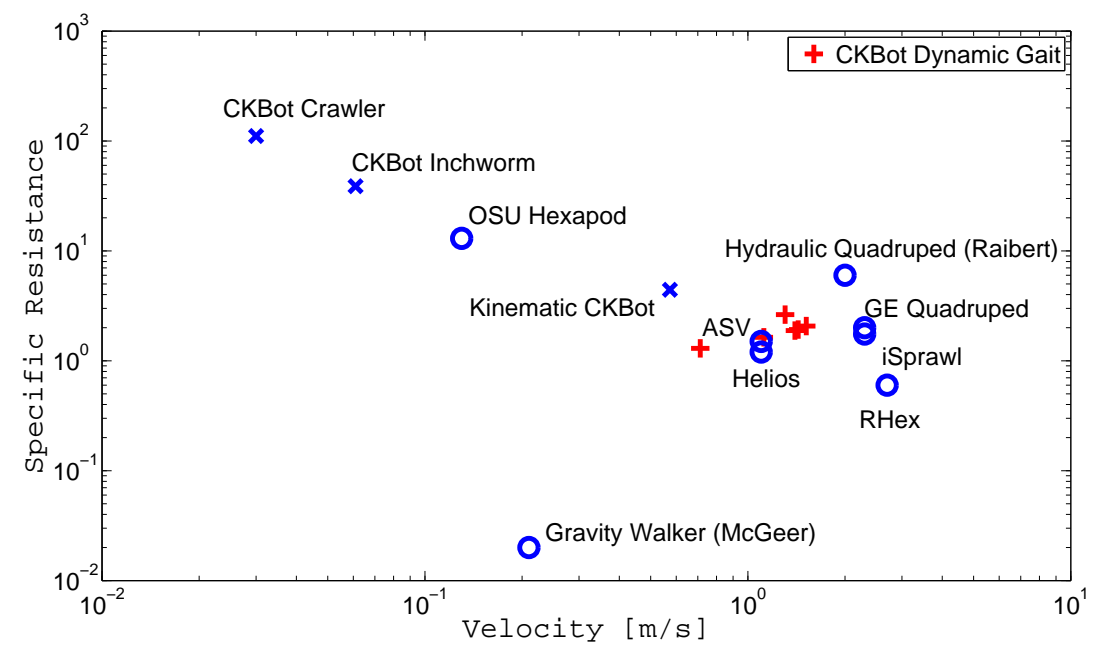

Fig. 16. Specific resistance vs. velocity for several robotic systems. The data for walking systems included here is from [8].

lower right corner of the graph. Robots shown in Figure 16 range from very energy efficient robots such as the Gravity Walker by McGeer [15] to very fast robots like RHex [19] and iSprawl [11].

It can be seen that the specific resistance for a dynamic rolling gait for CKBOT falls within a reasonable range of that for legged systems like RHex and iSprawl inspite of CKBOT's lack of powerful actuators. However, the larger number of actuators on CKBOT still raises the specific resistance substantially so that it is not as much lower than these fixed configuration non-wheeled robots, as would be expected.

Figure 16 also compares the dynamic rolling gait with other modular robot gaits. In Section VII, we saw that the dynamic rolling gait improved on the kinematic rolling gait. From Figure 16 it can be seen that it is a dramatic improvement on the inchworm gait and crawler gait. The inchworm gait was implemented with 10 CKBOT modules as well whereas the crawling gait was implemented with only 2 modules. These gaits are very slow and energy inefficient. The kinematic rolling gait is shown to have greater performance in terms of velocity and specific resistance, but it is the dynamic rolling gait that has pushed modular robots into the same range as walking systems.

The experimental results prove that this rolling gait is successful in traversing up and down inclines. Further, the trends in final measured terminal speeds for these cases match the expected trends, i.e. the robot rolls faster downhill than on level terrain and uphill.

We believe that the scaleability of the controller to configurations with different number of modules is a significant contribution of this work. It results from the choice of parameterization made for the controller and greatly reduces the computational complexity of scaling the controller. Thus, if a module or several modules break during a mission the system may continue after a simple reconfiguration discarding the failed modules. Conversely new modules can be picked up and added to the loop and the robot can keep going without having to expend significant resources to recompute control strategies.

The number of modules in the loop also has an effect on the performance of the rolling gait. A loop consisting of twice as many modules, with each module being half the length would have more joints, yet would retain its overall size in terms of length of the loop. As more joints are added to the loop the robot will more accurately approximate the arcs of the shape. The results show that making the arc less discrete will increase the velocity of the robot.

As the loop gets larger and larger the center of gravity of the robot gets higher. This should make the robot more susceptible to falling sideways in the sagittal plane. However, no significant instability in the sagittal plane was detected yet at a loop length of 14 modules in the case of CKBOT.

\section{Future WORK}

This work is part of a research effort to develop modular robots with a large number of modules and controllers that scale. In this context, we aim to develop controllers that can be easily adapted to a wide variety of modular robot configurations. The rolling loop configuration is the first step in this effort since it provides an easy and efficient mode of locomotion to cover large distances. Future effort in this direction will include the ability to turn and the exploration of optimization techniques to find optimal gaits. We also aim to examine dynamic controllers that allow the robot to adapt to rough terrain and locomote over unstructured environments using a conformal gait where the shape of the robot conforms to the profile of the ground in a dynamic fashion.

Figure 16 includes data for several robots that have improved on their specific resistance or terminal velocity using novel control and design techniques. iSprawl has a compliant leg design and achieves very fast speeds velocities and low specific 
resistance. Incorporating similar compliance into CKВОT will lessen the effect of impacts and improve its speed. Learning algorithms were used to tune the gaits for RHex [19] and resulted in almost a threefold increase in speed and a halving of specific resistance. These techniques could be adapted to rolling in order to achieve similar gains in both maximum speed and specific resistance. One strategy could be to use elongated shapes initially to achieve a high speed, then switch to a more energy efficient mode by using rounder shapes to maintain this speed.

The dynamic gait implemented in this work exploits the passive falling dynamics of the modular loop robot. Significant work has been performed in this area for walking robots [5], [6] where controllers are developed to take advantage of the passive dynamics of the robots to reduce torque requirements on the actuators. Indeed, McGeer's gravity walker [15] in Figure 16 has the lowest specific resistance amongst robots included in that Figure. Since modular robots have multiple actuated degrees of freedom, controllers that can reduce the requirements on the actuators would present significant benefits in extending the range and duration of operation of these systems.

The scalability of this controller addresses the interesting issue of scalable dynamics where models and controllers built for simpler systems can be easily adapted to larger systems. While this reduction to a simpler system was performed manually in our case, it might be possible to develop more general ideas for reducing complex configurations of modules to a simpler abstracted model for which controllers are easier to develop. Given the desire to ultimately extend this work to modular robots with hundreds or thousands of modules for which controllers would be extremely difficult to develop, the ability to abstract simpler models will play a significant role in being able to realistically deal with system of these sizes.

\section{REFERENCES}

[1] Z. Butler, K. Kotay, D. Rus, and K. Tomita. Generic decentralized control for a class of self-reconfigurable robots". In Proc. of the IEEE Int. Conf. on Robotics and Automation (ICRA'02), pages 809-815, Washington, D.C., 2002.

[2] A. Castano, W. M. Shen, and P. Will. Conro: Towards deployable robots with inter-robot metamorphic capabilities. Autonomous Robots Journal, 8(3):309-324, July 2000.

[3] G. S. Chirikjian. Kinematics of a metamorphic robotic system. In Proceedings of the IEEE International Conference on Robotics and Automation, pages $1452-1457,1996$.

[4] G.S. Chirikjian and J. W. Burdick. A modal approach to hyper-redundant manipulator kinematics. IEEE Transactions on Robotics and Automation, 10(3):343-354, June 1994.

[5] Steven H. Collins, Andy Ruina, Russ Tedrake, and Martjin Wisse. Efficient bipedal robots based on passive-dynamic walkers. Science, 307:1082-1085, 2005.

[6] Steven H. Collins, Martijn Wisse, and Andy Ruina. A three-dimensional passive-dynamic walking robot with two legs and knees. International Journal of Robotics Research, 20(7):607-615, 2001.

[7] Daniel Gomez-Ibanez, Ethan Stump, Ben Grocholsky, Vijay Kumar, and C. J. Taylor. The robotics bus: A local communications bus for robots. In Proceedings of the Society of Photo-Optical Instrumentation Engineers, 2004.

[8] Pedro Gregorio, Mojtaba Ahmadi, and Martin Buehler. Design, control and energetics of an electrically actuated legged robot. IEEE Transactions on Systems, Man and Cybernetics, 27(4):626-634, August 1997.

[9] A. Kamimura, H. Kurokawa, E. Yoshida, S. Murata, and K. Tomita. Automatic locomotion design and experiments for a modular robotic system. IEEE/ASME Transactions on Mechatronics, 10(3):314-325, June 2005.

[10] Akiya Kamimura, Satoshi Murata, Eiichi Yoshida, Haruhisa Kurokawa, Kohji Tomita, and Shigeru Kokaji. Self-reconfigurable modular robot - experiments on reconfiguration and locomotion. In Proceedings of 2001 IEEE/RSJ International Conference on Intelligent Robots and Systems (IROS200), pages 606-612, Maui, Hawaii, October 2001.

[11] Sangbae Kim. isprawl: Design and tuning for high-speed autonomous open-loop running. International Journal of Robotic Research, 25(9):903-912, September 2006.

[12] A. D. Kuo, J. M. Donelan, and A. Ruina. Energetic consequences of walking like an inverted pendulum: Step-to-step transitions. Exercise and Sport Science Review, 33(2), 2005.

[13] Woo Ho Lee and Arthur Sanderson. Dynamic rolling of modular robots. In Proceedings of the IEEE International Conference on Robotics and Automation, San Francisco, April 2000.

[14] T. Matsuda and S. Murata. Stiffness distribution control - locomotion of closed link robot with mechanical softness. In Proc. IEEE Int. Conf. Robotics and Automation, pages 1491-1498, Orlando, Florida, May 2006.

[15] Tad McGeer. Passive dynamic walking. Int. J. of Robotics Research, 9(2):62-82, April 1990.

[16] M. Park, S. Chitta, A. Teichman, and M. Yim. Automatic configuration recognition methods in modular robots. Submitted to IJRR, November 2006.

[17] Wei-Min Shen, Maks Krivokon, Harris Chiu, Jacob Everist, Michael Rubenstein, and Jagadesh Venkatesh. Multimode locomotion via superbot robots. In Proc. IEEE Int. Conf. Robotics and Automation, pages 2552-2557, Orlando, Florida, May 2006.

[18] Y. Sugiyama, A. Shiotsu, M. Yamanaka, and S. Hirai. Circular/spherical robots for crawling and jumping. In Proc. of the IEEE Int. Conf. on Robotics and Automation, Barcelona, Spain, April 2005.

[19] Joel D. Weingarten, Gabriel D. Lopes, Martin Buehler, Richard E. Groff, and Daniel E. Koditschek. Automated gait adaptation for legged robots. In Proceedings of the 2005 IEEE International Conference on Robotics and Automation, New Orleans, LA, April 2004.

[20] M. Yim. Locomotion With A Unit-Modular Reconfigurable Robot. PhD thesis, Stanford University, Palo Alto, CA, December 1994.

[21] M. Yim, C. Eldershaw, Y. Zhang, and D. G. Duff. Limbless conforming gaits with modular robots. In Proc. of the International Symposium on Experimental Robotics, Singapore, 2004.

[22] M. Yim, R. Hinden, C. Conley, C. K. Wang, K. D. Roufas, and C. Eldershaw. Open loop climbing with modular robots. In Video Proc. of the IEEE Intl. Conf. on Robotics and Automation, Orlando, FL, 2006.

[23] M. Yim, S. B. Homans, and K. D. Roufas. Climbing with snake-like robots. In IFAC Workshop on Mobile Robot Technology, Jejudo, Korea, MAY 2001.

[24] Y. Zhang, C. Eldershaw, M. Yim, K. D. Roufas, and D. G. Duff. A platform for studying locomotion systems: Modular self-reconfigurable robots. In Proc. of NIST workshop on Performance Metrics for Intelligent Systems (PerMIS), pages 893-899, Gaithersburg, MD, August 2002. 


\section{APPENDIX}

$$
\begin{gathered}
\ddot{\theta}_{g}=-\left(m g l\left(10 \cos \left(\theta_{g}\right)+11 \cos \left(\theta_{s}+\theta_{g}\right)+20 \cos \left(\frac{\theta_{s}}{2}\right) \cos \left(\left(5 \theta_{s}\right) / 2+\theta_{g}\right)\right)+2 \tau\right) / \\
\left(4\left(4 I_{0}+9 m l^{2}+2 m l^{2}\left(6 \cos \left(\theta_{s}\right)+3 \cos \left(2 \theta_{s}\right)+\cos \left(3 \theta_{s}\right)\right)\right)\right) \\
\ddot{\theta}_{a}=\left(m g l \left(\left(4 I_{0}+9 l^{2} m\right)\left(10 \cos \left(\theta_{g}\right)+11 \cos \left(\theta_{s}+\theta_{1}\right)+20 \cos \left(\theta_{s} / 2\right) \cos \left(\left(5 \theta_{s}\right) / 2+\theta_{g}\right)\right)\right.\right. \\
\left.-40\left(4 I_{0}+9 l^{2} m+2 l^{2} m\left(6 \cos \left(\theta_{s}\right)+3 \cos \left(2 \theta_{s}\right)+\cos \left(3 \theta_{s}\right)\right)\right) \cos \left(3 \theta_{s}+\theta_{g}+\theta_{a}\right)\right)+ \\
\left.2\left(5\left(4 I_{0}+9 l^{2} m\right)+8 l^{2} m\left(6 \cos \left(\theta_{s}\right)+3 \cos \left(2 \theta_{s}\right)+\cos \left(3 \theta_{s}\right)\right)\right) \tau\right) /\left(4\left(4 I_{0}+9 l^{2} m\right)\left(4 I_{0}+9 l^{2} m+2 l^{2} m\left(6 \cos \left(\theta_{s}\right)+3 \cos \left(2 \theta_{s}\right)+\cos \left(3 \theta_{s}\right)\right)\right)\right) ;
\end{gathered}
$$

$$
\ddot{\theta}_{s}=0 .
$$

